\title{
The Effect of Adiabatic Obstacles on Natural Convection inside a Horizontal Elliptical Porous Annulus
}

\author{
Faysal Ayad Khaleel ${ }^{1} \&$ Amir Sultan Dawood ${ }^{1}$ \\ ${ }^{1}$ Mechanical Engineering Department, Collage of Engineering, Mosul University, Mosul, Iraq \\ Correspondence: Faysal AyadKhaleel, Mechanical Engineering Department, Mosul University, Mosul, Iraq. Tel: \\ 964-780-231-5745. E-mail: f_eng23@yahoo.com
}

Received: May 27, 2013

Accepted: July 8, 2013 Online Published: August 6, 2013

doi:10.5539/mer.v4n1p1

URL: http://dx.doi.org/10.5539/mer.v4n1p1

\begin{abstract}
This study numerically examines the influence of installing two identical radial adiabatic obstacles on natural convection within a porous layer of constant thickness. This porous layer surrounds an isothermal hot elliptical cylinder and is surrounded by an isothermal cold one.The obstacles' length, angular position and radial position were changed in various amounts. A non-orthogonal curvilinear coordinate system is adopted, in order to treat the presence of the obstacles inside a two-dimensional elliptical annulus. Consequently, Finite Differences Techniques and ADI with Relaxation Method were used to approximate the computational regime of the non-Darcian-Boussinesq equation, governing the flow inside the computational porous domain. An efficient code of high accuracy was constructed to solve the approximated governing equations. The results showed that the installation of long obstacles in the meeting place of two convection cells causes an increase in heat transfer rates by up to $16 \%$. Whereas the installation of short obstacles in a place lying close to the center of the major convection cell causes a reduction in heat transfer by up to $19 \%$.
\end{abstract}

Keywords: porous media, elliptical annulus, non-darcian model, natural convection, elliptic grid generation, obstructions

\section{Nomenclature}

a Half of the major axis, $\mathrm{m}$

$b \quad$ Half of the minor axis, $\mathrm{m}$

Da Darcy number

$g \quad$ Gravitational acceleration, $\mathrm{m} \mathrm{s}^{-2}$

$J \quad$ The jacobian $\left(=\hat{x}_{\xi} \hat{y}_{\eta}-\hat{x}_{\eta} \hat{y}_{\xi}\right)$

$K \quad$ Permeability of porous medium, $\mathrm{m}^{2}$

L Length of the studied model (characteristic length), m

$\mathrm{Nu}$ Local Nusselt number

$\mathrm{P}$ Pressure, $\mathrm{Pa}$

Pr Prandtl number

$\mathrm{Ra}$ Rayleigh number

$\mathrm{Ra}^{*}$ Modified Rayleigh number

$\mathrm{T}$ Temperature, $\mathrm{K}$

$u$ Horizontal velocity component, $\mathrm{m} \mathrm{s}^{-1}$

$v \quad$ Vertical velocity component, $\mathrm{m} \mathrm{s}^{-1}$

v Velocity vector, $\mathrm{m} \mathrm{s}^{-1}$

\section{Accents}

$\widehat{T} \quad$ Non-dimensional

$\overline{\mathrm{Nu}}$ Average

\section{Greek symbols}

$\alpha \quad$ Thermal diffusivity, $\mathrm{m}^{2} \mathrm{~s}^{-1}$

$\beta \quad$ Thermal expansion coefficient, $\mathrm{K}^{-1}$

$\gamma=\hat{\mathrm{x}}_{\xi}{ }^{2}+\hat{\mathrm{y}}_{\xi}^{2}$

$\delta=\hat{\mathrm{x}}_{\xi} \hat{\mathrm{x}}_{\eta}+\hat{\mathrm{y}}_{\xi} \hat{\mathrm{y}}_{\eta}$

$\varepsilon \quad=\hat{\mathrm{x}}_{\eta}{ }^{2}+\hat{\mathrm{y}}_{\eta}{ }^{2}$

$\mu \quad$ Dynamic viscosity, Pa s

$v \quad$ Kinematic viscosity, $\mathrm{m}^{2} \mathrm{~s}^{-1}$

$\xi, \eta \quad$ Computational region coordinates

$\rho \quad$ Density, $\mathrm{kg} \mathrm{m}^{3}$

$\phi \quad$ Porosity of porous medium

$\psi \quad$ Stream function, $\mathrm{m}^{2} \mathrm{~s}^{-1}$

$\omega \quad$ Vorticity, $s^{-1}$

\section{Subscripts}

c Cold surface

$f \quad$ fluid

h Hot surface

$i \quad$ Inner cylinder surface

$o \quad$ outer cylinder surface 


\section{Introduction}

Natural convection heat and mass transfer in porous media has been studied extensively. This is due to the increasing need to understand the complicated transport process for application of diverse insulation fields which include insulation of hot or cold pipes, aircraft cabin, nuclear and power plants and solar collector receivers. Moreover, the porous media are used or naturally exist in fields rather than those of insulation. For example, geothermal engineering, solid matrix heat exchanger (compact heat exchanger), oil extraction, underground disposal of nuclear waste materials, and many more.

A special case study of natural convection inside porous media is that medium bounded by two concentric (or eccentric) cylinders. The previous published studies of such case have demonstrated, both experimentally and theoretically, some details related to the nature of fluid flow and heat transfer inside porous media. Bishop and Charley (1966) investigated the phenomenon of natural convection in a porous layer put between two concentric horizontal cylinders. The results photographically showed the fluid motion inside the enclosure. Another study on the same geometrical shape and boundary conditions had been both experimentally and theoretically done by Caltagirone (1976). He investigated the axial $\left(3^{\text {rd }}\right)$ direction heat transfer and concluded that there is a critical value for the modified Rayleigh number, across which the $3^{\text {rd }}$ direction flow is undergoing taking place.Bau et al. (1983) carried out the experiment of Caltagirone again with two eccentric cylinders. They observed the growth of the multicellular in the upper part of the model. This multicellular had gradually transformed to a unicellular as time passes. Charrier and Mojtabi (1991) had also studied the free convection (2D) flow in a horizontal saturated porous annulus. They joined their numerical study with an experimental work. Their work led to conclude that the transition from unicellular to multicellular flow depends strongly on the initial conditions. Moreover, they concluded that the heat transferred in the axial direction is an unsteady state phenomenon. Sakr et. al., (2008) experimentally and numerically studied the natural convection inside an annulus bounded by inner elliptical tube and outer circular one. Their results showed that the horizontal major axis position gives more efficient insulation than the vertical position. Mota and Saatdjian $(1995,1999)$ numerically studied the hysteresis loop inside a circular and elliptical annuli respectively. They concluded that the behavior of the flow and (Ra-Nu) curve depend on the initial thermal condition. Another study for Mota et al. (2000) concluded that the judicious horizontal stretching of one of the annulus's boundaries reduces heat losses with respect to concentric cylindrical annulus with the same amount of insulating material. Zhu et al. (2003) also numerically investigated the effect of the major axis position of the elliptic boundaries. They concluded that more vortices in streamlines is generated in the case of horizontal position. Moreover, they did mention that further investigations may be needed for the phenomenon to be clear and understood.

All the aforementioned theoretical studies used the Darcian model to describe the momentum equation of the flow. However, Kaviany (1986) assumed a circular annulus and studied the effect of the inertia, boundary and velocity-square terms. His results showed that all of these effects reduces heat losses with the boundary term being the most significant. Moreover, an acceptable agreement between the Darcian and non-Darcian regimes holds for low Rayleigh and Prandtl numbers. Braga and de Lemos (2006) had a circular porous annulus and numerically simulated the turbulent natural convection by using a macroscopic non-Darcian two-equation model. They studied the dependency of the laminarization phenomenon on Darcy number and porosity.

The effect of inserting an obstacle inside a rectangular, heated-cooled and porous cavity was studied by Bejan (1983). The results indicated an increase in heat losses in some cases of the horizontal obstacle's length. On the other hand, a vertical obstacle of $l / H=1$ decreases the heat lost. Facas (1994) numerically tried to reduce the heat transfer from a hot pipe buried in a semi-infinite, saturated, porous medium, by installing two obstacles on the pipe's wall. The results indicated a maximal energy saving in the case of horizontal radial position.

In the present study the effect of inserting two radial adiabatic obstacles on natural convection within a porous layer of a constant thickness was numerically examined. These two obstacles were symmetrically set about the vertical axis of the study's model with various amounts of length and angular position. The porous layer surrounds an isothermal hot elliptical cylinder and surrounded by an isothermal cold one. This study also assumed the 2-D non-Darcian model with Boussinesq approximation to represent the momentum equation governing the flow within the porous medium.

\section{Mathematical Formulation}

The physical problem of the present study is considered by a two-dimensional elliptical cross section, as illustrated in Figure 1. This 2D annulus is completely defined by the following equations and parameters. Firstly, the following equation generates the inner boundary of the annulus: 


$$
\left(\frac{\mathrm{x}_{i}}{a_{i}}\right)^{2}+\left(\frac{\mathrm{y}_{i}}{b_{i}}\right)^{2}=1
$$

The hydraulic radius ratio $(\mathrm{R})$ is given as follows:

$$
\mathrm{R}=\sqrt{a_{o} b_{o}} / \sqrt{a_{i} b_{i}}
$$

The thickness $(t)$ of the porous layer is kept constant in each angular position and considered as a function of (R). So, it is given by:

$$
t=\sqrt{\left(\frac{a_{i}+b_{i}}{2}\right)^{2}+a_{i} b_{i}\left(\mathrm{R}^{2}-1\right)}-\left(\frac{a_{i}+b_{i}}{2}\right)
$$

For the thickness $(t)$ being constant, the annulus's outer boundary is given by:

$$
\begin{gathered}
\mathrm{x}_{\mathrm{o}}=\mathrm{x}_{\mathrm{i}}+\mathrm{t} \cdot \sin (180-\varphi) \\
\mathrm{y}_{\mathrm{o}}=\mathrm{y}_{\mathrm{i}}+\mathrm{t} \cdot \cos (180-\varphi)
\end{gathered}
$$

Where $(\varphi)$ is the local slope angle of the inner boundary curve.

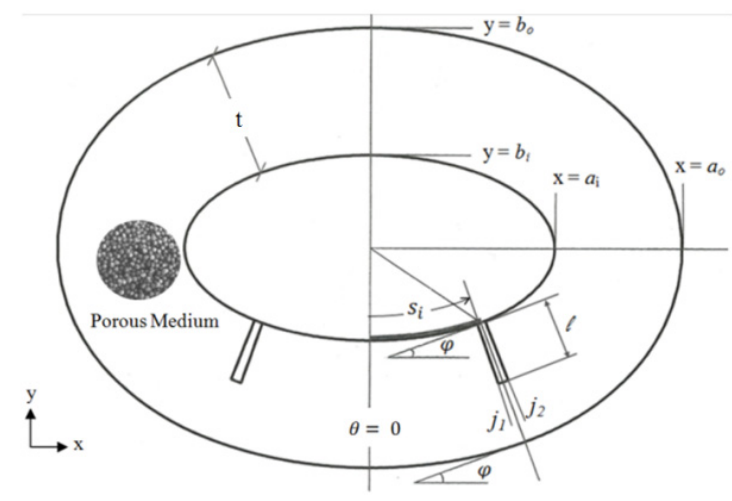

Figure 1. Geometrical configuration of the present study

The obstacles are set normal to the tangent of the boundary curvature as illustrated in Figure 1. Their length $(l)$ are given with respect to the porous layer thickness $(t)$. The angular position $(\theta)$ is described by the ratio of the circumferential length $\left(s_{i}\right)$ to the whole length of the circumference $\left(c_{i}\right)$, as the following:

$$
\theta=\frac{s_{i}}{C_{i}} \times 360^{\circ}
$$

Where:

$$
\begin{gathered}
s_{i}=\int_{0}^{\mathrm{x}_{i}} \sqrt{\left(\frac{d \mathrm{y}_{i}}{d \mathrm{x}_{i}}\right)^{2}+1} d \mathrm{x}_{i} \\
c_{i}=4 \int_{0}^{a_{i}} \sqrt{\left(\frac{d \mathrm{y}_{i}}{d \mathrm{x}_{i}}\right)^{2}+1} d \mathrm{x}_{i}
\end{gathered}
$$

The present study assumed steady state, laminar and incompressible flow, with homogenous saturated porous medium of non-conductive solid matrix. The annulus is of isothermal boundaries, and the inner is hot $\left(\mathrm{T}_{\mathrm{h}}\right)$, whereas the outer is cold $\left(\mathrm{T}_{\mathrm{c}}\right)$. The solid matrix is in thermal equilibrium with the fluid flowing inside it, and no viscous dissipation is assumed. The thermo-physical properties are kept constant except for density variation in the buoyancy force term (Boussinesq approximation).

According to the aforementioned assumptions and considerations, the equations govern the steady-state two-dimensional problem in Cartesian coordinates will be as follows (Bejan, 1995; Vafai, 2005): 


\section{Continuity}

Momentum

$$
\frac{\partial u}{\partial \mathrm{x}}+\frac{\partial v}{\partial \mathrm{y}}=0
$$

$$
\frac{\partial \mathrm{P}}{\partial \mathrm{x}}=-\frac{\mu}{K} u-\frac{\rho_{f} \mathrm{~F}}{\sqrt{K}} u|\mathbf{v}|+\mu \nabla^{2} u-\frac{\rho_{f}}{\emptyset^{2}}(\mathbf{v} . \nabla) u
$$

and

$$
\frac{\partial \mathrm{P}}{\partial \mathrm{y}}=-\frac{\mu}{K} v-\frac{\rho_{f} \mathrm{~F}}{\sqrt{K}} v|\mathbf{v}|+\mu \nabla^{2} v-\frac{\rho_{f}}{\emptyset^{2}}(\mathbf{v} \cdot \nabla) v+\rho_{f} \mathbf{g}
$$

Energy

$$
\nabla^{2} \mathrm{~T}=\frac{1}{\alpha_{f}}(\mathbf{v} \cdot \nabla) \mathrm{T}
$$

Where $\mathrm{F}$ is the non-dimensional form of the drag constant defined by the Formula (13) used by Hadim and North, (2005).

$$
\mathrm{F}=\frac{1.75}{\sqrt{150}} \times \emptyset^{-3 / 2}
$$

The non-dimensional forms of the physical quantities and variables, included in the above equations or needed in transforming them to their dimensionless forms, are defined as the following:

$$
\begin{gathered}
\hat{\mathrm{x}}=\frac{\mathrm{x}}{\mathrm{L}}, \hat{\mathrm{y}}=\frac{\mathrm{y}}{\mathrm{L}}, \hat{\mathrm{a}}=\frac{\mathrm{a}}{\mathrm{L}}, \hat{\mathrm{b}}=\frac{\mathrm{b}}{\mathrm{L}}, \widehat{\mathrm{P}}=\frac{\mathrm{PL}^{2}}{\rho_{f} \alpha_{f}{ }^{2}}, \widehat{\omega}=\frac{\omega \mathrm{L}^{2}}{\alpha_{f}}, \hat{u}=\frac{u \mathrm{~L}}{\alpha_{f}}, \hat{v}=\frac{v \mathrm{~L}}{\alpha_{f}}, \hat{\psi}=\frac{\psi}{\alpha_{f}}, \\
\mathrm{Ra}=\frac{\mathrm{g} \beta\left(\mathrm{T}_{\mathrm{h}}-\mathrm{T}_{\mathrm{c}}\right) \mathrm{L}^{3}}{v \alpha_{f}}, \mathrm{Da}=\frac{K}{\mathrm{~L}^{2}}, \mathrm{Ra}^{*}=\mathrm{Ra} \cdot \mathrm{Da}, \operatorname{Pr}=\frac{v}{\alpha_{f}}, \widehat{\mathrm{T}}=\frac{\mathrm{T}-\mathrm{T}_{\mathrm{c}}}{\mathrm{T}_{\mathrm{h}}-\mathrm{T}_{\mathrm{c}}}
\end{gathered}
$$

The pressure terms in Equations (10) and (11) can be eliminated by differentiating these equations with respect to (y) and (x) respectively, and then subtracting one from other. Hereafter, by using forms (14), the dimensionless governing equations in terms of vorticity and stream function, will respectively be as follows (Kaviany, 1986):

$$
\begin{aligned}
& \widehat{\omega}=-\widehat{\nabla}^{2} \widehat{\psi} \\
& \widehat{\omega}-\operatorname{Da} \cdot \widehat{\nabla}^{2} \widehat{\omega}=\operatorname{Ra}^{*} \frac{\partial \widehat{T}}{\partial \hat{\mathrm{x}}}+\frac{\mathrm{F} \cdot \sqrt{\mathrm{Da}}}{\operatorname{Pr}}\left\{\hat{u} \frac{\partial|\hat{\mathbf{v}}|}{\partial \hat{\mathrm{y}}}-\hat{v} \frac{\partial|\hat{\mathbf{v}}|}{\partial \hat{\mathrm{x}}}\right\}-\frac{\mathrm{F} \cdot \sqrt{\mathrm{Da}} \cdot|\hat{\mathbf{v}}|}{\operatorname{Pr}} \widehat{\omega}-\frac{\mathrm{Da}}{\operatorname{Pr} \cdot \emptyset^{2}}(\hat{\mathbf{v}} \cdot \widehat{\nabla}) \widehat{\omega} \\
& \widehat{\nabla}^{2} \widehat{T}=(\hat{v} \cdot \widehat{\nabla}) \widehat{T}
\end{aligned}
$$

Where $(\hat{\mathrm{u}}=\partial \widehat{\psi} / \partial \hat{\mathrm{y}})$ and $(\hat{\mathrm{v}}=-\partial \widehat{\psi} / \partial \hat{\mathrm{x}})$.

The appropriate boundary conditions, in their non-dimensional forms, are listed below:

1) Inner cylinder:

$$
\widehat{\mathrm{T}}=1, \quad \hat{\psi}=0, \quad \hat{u}=0, \quad \hat{v}=0, \quad \widehat{\omega}=-\frac{\partial^{2} \hat{\psi}}{\partial \hat{\mathrm{n}}^{2}}
$$

2) Outer cylinder :

$$
\widehat{\mathrm{T}}=0, \widehat{\psi}=0, \hat{u}=0, \hat{v}=0, \quad \widehat{\omega}=-\frac{\partial^{2} \hat{\psi}}{\partial \hat{\mathrm{n}}^{2}}
$$

3) Obstacles' surfaces:

$$
\frac{\partial \widehat{\mathrm{T}}}{\partial \hat{\mathrm{n}}}=0, \hat{\psi}=0, \hat{u}=0, \hat{v}=0, \widehat{\omega}=-\frac{\partial^{2} \hat{\psi}}{\partial \hat{\mathrm{n}}^{2}}
$$

Where $\hat{n}$ is the non-dimensional local normal vector, and:

$$
\frac{\partial}{\partial \hat{\mathrm{n}}}=\sqrt{\left(\frac{\partial}{\partial \hat{\mathrm{x}}}\right)^{2}+\left(\frac{\partial}{\partial \hat{\mathrm{y}}}\right)^{2}}
$$


A non-orthogonal curvilinear coordinate system is adopted, in order to treat the irregular boundaries of the studied elliptical annulus. Consequently, transformations from the physical regime, which is Cartesian, to a computational regime of $(\xi, \eta)$ coordinates are performed by assuming $\xi=\xi(\hat{x}, \hat{y})$ and $\eta=\eta(\hat{x}, \hat{y})$.

The grid of this study, was generated by assuming elliptic Poisson's equations of a control method adopted by Hoffmann and Chiang (2000). The following partial differential equations were solved to obtain the desired grid:

$$
\begin{aligned}
& \varepsilon\left(\hat{\mathrm{x}}_{\xi \xi}+\Phi \hat{\mathrm{x}}_{\xi}\right)-2 \delta \hat{\mathrm{x}}_{\xi \eta}+\gamma\left(\hat{\mathrm{x}}_{\eta \eta}+\Psi \hat{\mathrm{x}}_{\eta}\right)=0 \\
& \varepsilon\left(\hat{\mathrm{y}}_{\xi \xi}+\Phi \hat{\mathrm{y}}_{\xi}\right)-2 \delta \hat{\mathrm{y}}_{\xi \eta}+\gamma\left(\hat{\mathrm{y}}_{\eta \eta}+\Psi \hat{\mathrm{y}}_{\eta}\right)=0
\end{aligned}
$$

Where $\Psi$ and $\Phi$ are respectively defined as:

$$
\begin{gathered}
\Psi=-\left.\frac{\hat{\mathrm{x}}_{\eta \eta} \hat{\mathrm{x}}_{\eta}+\hat{\mathrm{y}}_{\eta \eta} \hat{\mathrm{y}}_{\eta}}{\hat{\mathrm{x}}_{\eta}{ }^{2}+\hat{\mathrm{y}}_{\eta}{ }^{2}}\right|_{\xi=\text { constant }} \\
\Phi=-\left.\frac{\hat{\mathrm{x}}_{\xi \xi} \hat{\mathrm{x}}_{\xi}+\hat{\mathrm{y}}_{\xi \xi} \hat{\mathrm{y}}_{\xi}}{\hat{\mathrm{x}}_{\xi}{ }^{2}+\hat{\mathrm{y}}_{\xi}{ }^{2}}\right|_{\eta=\text { constant }}
\end{gathered}
$$

Suitable clustering functions were used for raising the grid resolution in some places inside the studied region, where the physical variables are of highly stepper gradient (Petrovic \& Stupar, 1996). Figure 2 illustrates the resulted grid.

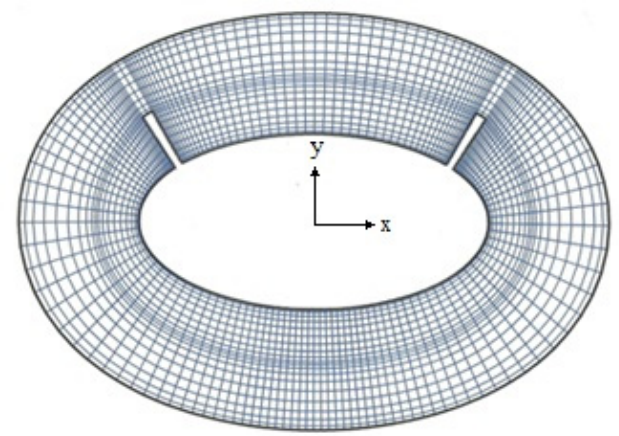

Figure 2. Schematic sketch of the computational grid generated by Poisson's elliptic equation

The local heat transfer at the inner and outer surfaces are represented by the local Nusselt number. And the latter is expressed in computational regime as the following (Zhu et al., 2004):

$$
\mathrm{Nu}=-\frac{\sqrt{\gamma}}{J}\left(\frac{\partial \widehat{\mathrm{T}}}{\partial \eta}\right)
$$

The average Nuselt number is then determined by integrating the local Nusselt number along the surface of the inner or outer cylinder, taking in consideration the elimination of the adiabatic obstacles' bases from calculations.

\section{Numerical Procedure}

After the governing differential equations, with the appropriate boundary conditions, had been transformed to the computational regime $(\xi, \eta)$, they were approximated utilizing finite differences techniques of second order truncation error. The approximated equations were solved numerically by using ADI with under relaxation method. The relaxation factor depended on the geometric variables. So, it had different values, each is determined by trial and error. However, the optimal under relaxation factor in all cases was less than unity.

The independence of the numerical results from the grid size was also verified by trial and error. $(49 \times 200)$ was the chosen size, with which, the energy balance was, at worst, preserved to less than $1.6 \%$ in all calculations.

The convergence criterion, adopted in this study, was defined as follows:

$$
\left|\frac{\Theta^{\tau+1}-\Theta^{\tau}}{\Theta^{\tau+1}}\right| \leq 10^{-6}
$$

Where $\Theta$ is any local dependent variable and $\tau$ is the number of the last iteration. On the other hand, the 
maximum number of iterations depended on the values of the relaxation factor. The deeper the latter, the more the former needed to verify the error set. It is worth to mention that Matlab programming language was utilized to construct the computer code which generated the numerical results.

\section{Code Validation}

In order to validate the code, several previous works should be accommodated by resetting the input variables of the geometry and porous medium. Figure 3 shows the streamlines and isotherms obtained by the present study in compare with those of Braga and de Lemos (2006), for ( $\mathrm{Da}=3 \times 10^{-7}, \emptyset=0.2, \mathrm{Ra}^{*}=500 \& \mathrm{R}=2$ ).
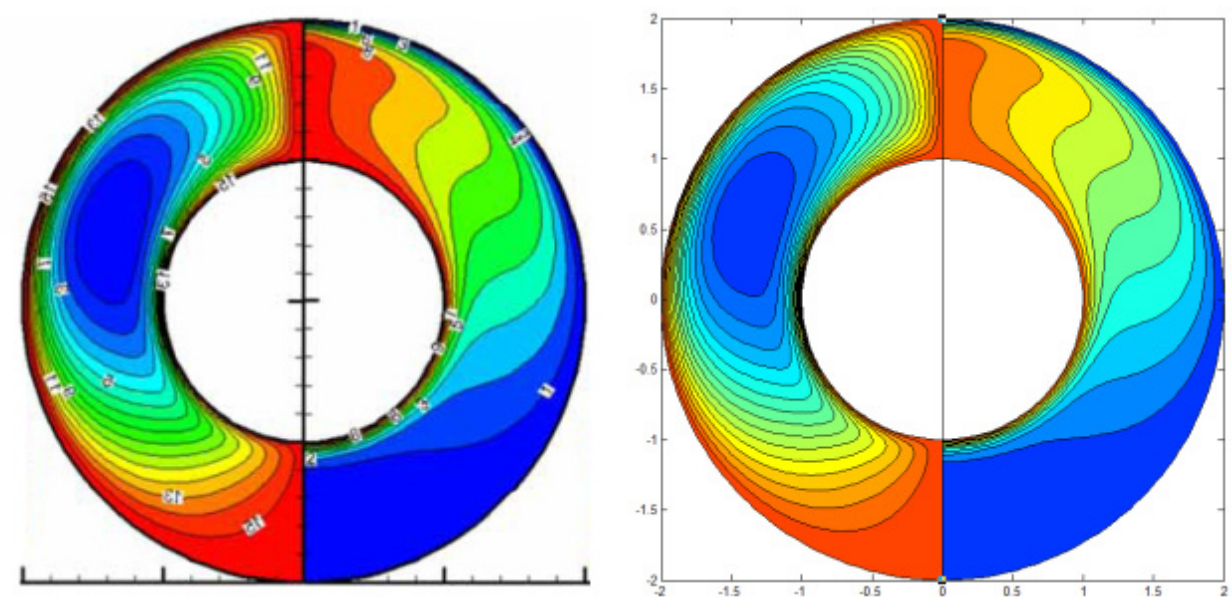

Figure 3. Streamlines and isotherms' comparison of the present study (to the right) and Braga-do Lemos, (2006) (to the left) for $\left(\mathrm{Da}=3 \times 10^{-7}\right),(\varnothing=0.2),\left(\mathrm{Ra}^{*}=500\right)$ and $(\mathrm{R}=2)$

The results of the average Nusselt number, obtained by the code of the present study, were also validated for a range of modified Rayleigh number. So, Figure 4 shows a comparison between the results of the present study and those experimentally and theoretically obtained by some previous works. Regarding the presence of the obstacles inside porous annuli, no comparable study is available up to the authors' knowledge.

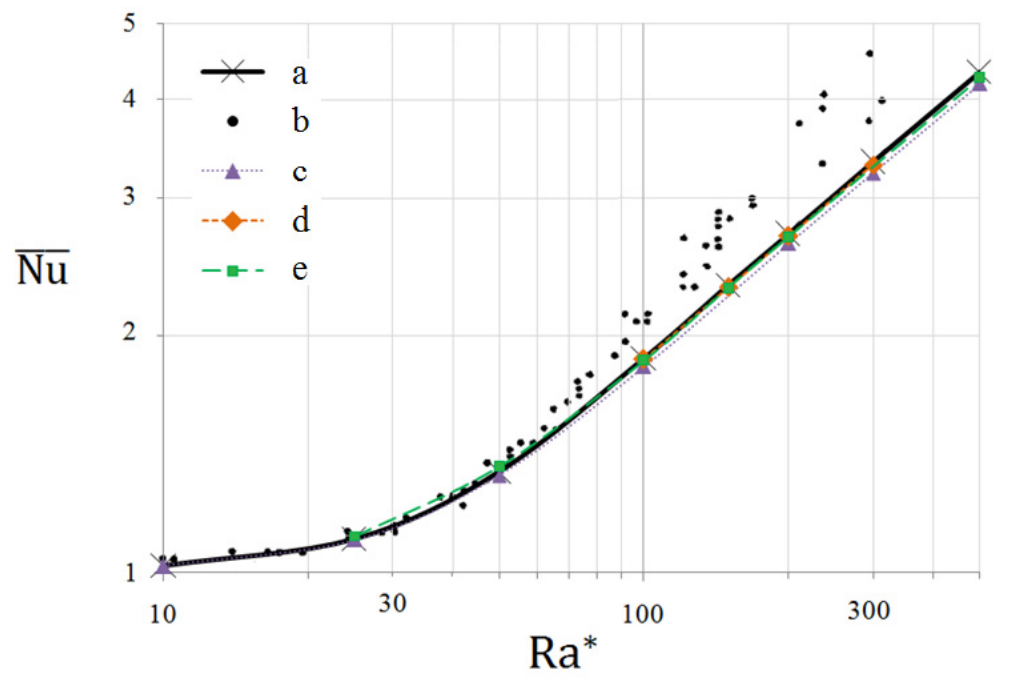

Figure 4. Average Nusselt number versus modified Rayleigh number for $\left(\mathrm{Da}=3 \times 10^{-7}\right),(\varnothing=0.2)$ and $(\mathrm{R}=$ 2). (a): Present work, (b): Experimental (Caltagirone, 1976), (c): Numerical (Caltagirone, 1976), (d): Unicellular flow (Charrier-Mojtabi et. al., 1991) and (e): Laminar model (Braga-de Lemos, 2006)

Performing the above comparisons indicated a good agreement with the previous works and ensured that the constructed code is reliable enoughto solve the problem of the present work. 


\section{Results and Discussion}

For the purpose of simplifying the presentation of geometric cases, a special symbolic system is adopted in this study. For example, $(0.67-\mathrm{PI}-90)$ means that the twin obstacles are of length $(l=0.66 t)$, and they are fixed on the surface of the inner cylinder at $\left(\theta=90^{\circ}\right)$. Within this code, (PI) would be replaced by (PO), if the twin obstacles were fixed on the outer cylinder. However, this study examines the influence of the obstacles' existence by assuming three angular positions $\left(60^{\circ}, 90^{\circ}\right.$ and $\left.120^{\circ}\right)$, two radial positions (PI and PO) and three lengths $(0.33$, 0.5 and 0.66 ).

The influence of modified Rayleigh number in porous annuli has already been investigated in details by many authors (e.g.: Caltagirone, 1976, Charrier-Mojtabi, 1991, Mota \& Saatdjian, 1995, 1999, 2000; Kaviany, 1986). So, in order to focus on the obstacles effect, the results discussed here are obtained at a single modified Rayleigh number of 500 and a single hydraulic radius ratio of 2:1. The combined effect of Rayleigh number, radius ratio and the obstacles would be all examined in other future studies. Darcy number, Prandtl number and porosity are also fixed for the same reason. They respectively are: $\left(10^{-4}, 0.71\right.$ and 0.9$)$.

The modified Rayleigh number 500 is high enough for the convective effects and the cellular flow pattern to be clear. Moreover, the installation of the obstacles is more needed and applicable at such a high Rayleigh number. Figure 5 shows a tri-cellular flow pattern occurring at $\left(\mathrm{Ra}^{*}=500\right)$ and $(\mathrm{R}=2)$. From this figure one can notice that the angular position $90^{\circ}$ is close to the center of the major convective cell where the maximum amount of stream function is. As so as, the direction of the flow, is almost normal to the obstacles, whether it is applied there. On another hand, the position $120^{\circ}$ is occupied by the meeting boundaries of two convection cells. Besides that, the direction of the flow there, is tangential to the obstacles. These two different positions were chosen by the authors to be occupied by their twin obstacles for the recently aforementioned reasons. In addition to the positions $90^{\circ}$ and $120^{\circ}$, the position $60^{\circ}$ was also studied in order to examine how the existence of the obstacles in the lower part of the model does affect the flow pattern and hence the heat transfer rate. Based on what was foregoing, the following sections covers all the mentioned cases.

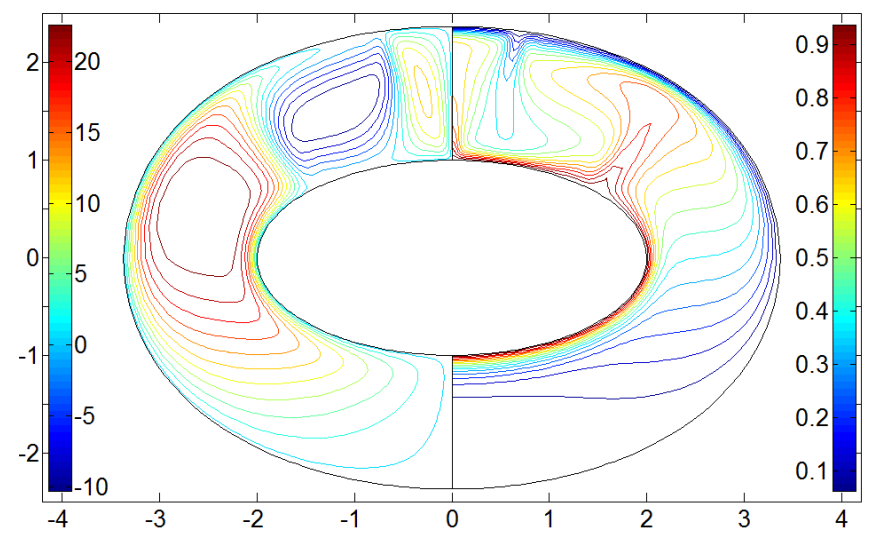

Figure 5. Streamlines (left) and isotherms (right) for $\mathrm{Ra}^{*}=500$ and $\mathrm{R}=2$, tri-cellular flow pattern without obstacles. It should be noticed that the clockwise flow direction has been negatively signed and vice versa

\section{a. The angular position $60^{\circ}$}

The aim of applying the obstacles in such a position is to confine and stagnate some of fluid under the inner cylinder and eliminate the convection currents there. The three length cases of PI-60 had quite performed that aim. The isotherms in Figures 6 ( $a, b$ and $c$ ) ensures that the heat almost conductively transfers in the lower part of the model. The other radial position's cases (PO-60), respectively shown in Figures 6 (d, e and f), hadn't do the same, but they just distorted the streamlines and slightly the cases of lengths and radial positions had already changed the flow pattern from tri-cellular to bi-cellular obstructed the major convection cells. On another hand, all.

Table 1 shows that the average Nusselt number had decreased by considerable and approximately equal rates. Whereas the decrease in the maximum stream function was clearly different between the two radial positions (PI and PO). All these observations lead to the conclusion that the presence of the obstacles in the lower part of the model reduces the heat transfer significantly by reducing the number of convection cells, not necessarily by obstructing the fluid or by confining it there. 
Table 1. Percentage of variation in the average Nusselt number and the maximum stream function

\begin{tabular}{llcccccc}
\hline & \multicolumn{2}{c}{$l=0.33$} & \multicolumn{2}{c}{$l=0.50$} & \multicolumn{2}{c}{$l=0.66$} \\
\hline \multirow{2}{*}{$45^{\circ}$} & PI & -08.20 & -5.73 & -08.12 & -5.38 & -08.07 & -5.15 \\
& PO & -08.71 & -2.8 & -11.04 & -2.27 & -07.94 & -1.85 \\
\multirow{2}{*}{$60^{\circ}$} & PI & -08.90 & -8.62 & -09.18 & -7.93 & -09.16 & -6.96 \\
& PO & -09.93 & -3.03 & -08.43 & -2.41 & -08.50 & -1.55 \\
$90^{\circ}$ & PI & -18.26 & -13.51 & -16.37 & -9.27 & -14.44 & -4.59 \\
& PO & -14.42 & -4.36 & -12.34 & -2.78 & -11.33 & -1.84 \\
$120^{\circ}$ & PI & +15.66 & -11.53 & +12.37 & -11.44 & +07.98 & -12.14 \\
& PO & -01.29 & -8.89 & +05.76 & -8.46 & +03.55 & -11.54 \\
$145^{\circ}$ & PI & -01.00 & -7.85 & -01.47 & -8.11 & -01.58 & -7.37 \\
& PO & -06.06 & -1.7 & -06.27 & -1.65 & -02.81 & -6.14 \\
\hline
\end{tabular}

$\left.\overline{N u}\right|_{\text {without obstacles }}=3.23$

$\left.\psi_{\text {max }}\right|_{\text {without obstacles }}=24.8$

\section{b. The angular position $\mathbf{9 0}^{\circ}$}

The presence of the obstacles according to case (0.33-PI-90) causes obstructing to the flow in a spot where the velocity was relatively high. Moreover, presence of the obstacles in that position, with any length, causes lengthening to the flow path by diverting it from the alignment of the hot cylinder, in that spot, to the alignment of the adiabatic obstacle's surface (see Figures 6 (g, h and i)). As a result, the decrease in the average Nusselt number is larger than the decrease in the maximum stream function, as it is clearly shown by Table 1. On another hand, the long (or moderate) obstacles, applied in the position (PI-90), partially separates the annulus into upper active part and lower inactive one. This separation causes a short circuit to the convection currents in the upper part. Consequently, such separation badly affects the insulation efficiency. This conclusion was supported by the results of Bejan (1983) exactly which concerning with full separation. Furthermore, Table 1 in the present study shows that the shorter the obstacles, the less the average Nusselt number in case of (PI-90). Summarily, the above conclusions may indicate an equilibrium, in which the effect of the obstructing and the effect of separation are on contrast.

Regarding the position (PO-90), the short obstacles, installed on the outer cylinder, obstructs the flow, but slightly less than what caused by installation in position (PI-90). The reason would be explained as follows: the side (major) convection cell expands along a curved enclosure. Although the installation of a short obstacle in position (PO-90) slightly chokes the flow, it reduces the severity of enclosure curvature. On contrast, installation in position (PI-90) increases the severity of enclosure curvature where the side convection cell expands along. On the other hand, installing long (or moderate) obstacles leads to a partial separation for the studied model into upper and lower parts. Figures $6(\mathrm{j}, \mathrm{k}$ and $\mathrm{l})$ show the posed cases in terms of isotherms and streamlines.

\section{c. The angular position $120^{\circ}$}

The position (PI-120) is considered the worst for the goal of reducing heat transfer. Table 1 shows an increase in Nusselt number up to $15.66 \%$ for some cases, despite of the drop in the maximum stream function. In order to understand the reason, the few following lines should be explicated firstly. The flow, within the small convection cells in the upper part, is subject to two opposite effects: the first is the contact with the major cell in the lower part, which directs the rotation of the adjacent upper cell to be negative. And this is actually what is taking place in case of no obstacle installed. The second is the effect of the geometry of the space including the upper convection cells. By looking at Figure 5, one can conclude that, if the moderate convection cell was left to spin under only the effect of geometry, it would have already taken the positive direction. This contrary in effect significantly reduces the amount of fluid flow in the upper part. Therefore, the installation of the obstacles, in the position (PI-120), increases the amount of flow in the upper part by creating small secondary cell at the obstacle head and eliminating the influence of contact with the major cell in thelower part. Consequently, this installation allows the upper main cell to spin freely in the positive direction under only the influence of the geometry of the space. 


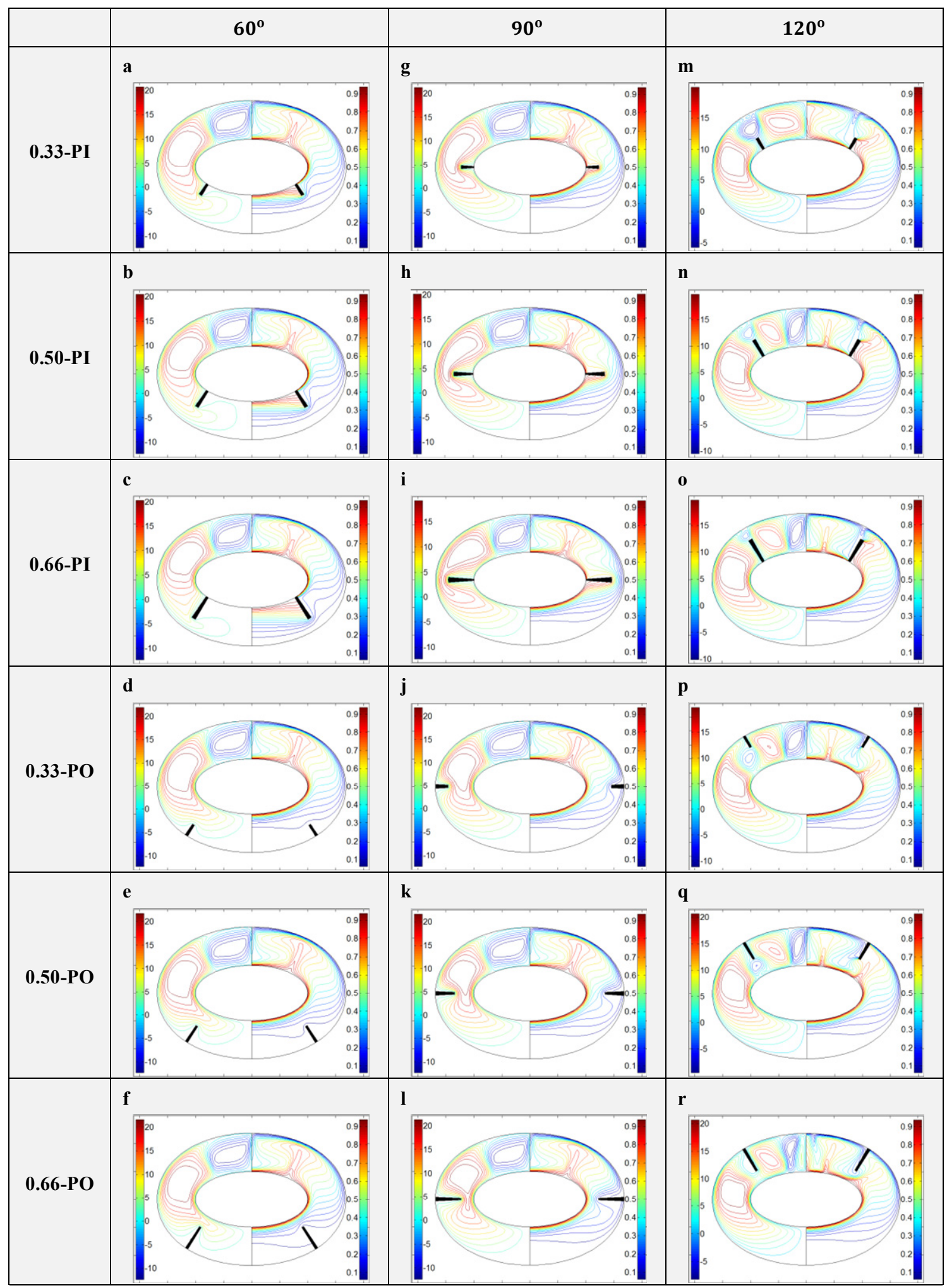

Figure 6. Streamlines (to the left) and isotherms (to the right) for various cases of angular position, radial position and length. $\left(\mathrm{Da}=10^{-4}\right),(\varnothing=0.9),\left(\mathrm{Ra}^{*}=500\right)$ and $(\mathrm{R}=2)$

All the foregoing above, leads to understand that the reduction in the maximum stream function, due to the presence of the obstacles, indicates reduction in the amount of fluid flow within the major cell. And this is regardless of the increase in the amount of the upper part flow, which is the actual reason of the increase in the 
overall average Nusselt number (see Table 1).

No fundamental difference exists between the two radial positions (PI-120 and PO-120) except the case (0.33-PO-120). Since, this case didn't cause enough separation. But on contrary, it caused a creation of a small additional convection cell of a significant effect on the major convection cell in the lower part. Table 1 shows that this case is unpractical to carry out.

\section{The Conclusions}

A non-orthogonal curvilinear coordinate system is adopted, in order to treat the presence of the obstacles inside two-dimensional elliptical annulus. Consequently, an efficient code of high accuracy has been developed to solve the non-Darcian-Boussinesq equation, governing the flow inside porous media. The code had already been validated then the results were discussed. Hereafter, several conclusions are listed below:

1) The presence of the obstacles in the lower part of the model reduces the heat losses significantly by changing the flow pattern and reducing the number of convection cells, not necessarily by obstructing the fluid or by confining it there.

2) When the obstacles is installed in the angular position $90^{\circ}$, the shorter the obstacles, the less the overall heat losses. However, the installing at the inner cylinder, in such an angular position, reduces the heat losses more than the reduction caused in case of installing the obstacles at the outer cylinder.

3) The best position for the obstacles to be installed in, with respect to the goal of reducing heat losses, is $90^{\circ}$. Whereas the worst is $120^{\circ}$. So, in such a problem, one can preserve $18.26 \%$ of the lost energy, while another may lose $15.66 \%$ of it.

\section{Acknowledgements}

The authors acknowledge reconcile of God, besides to the support of Mechanical Engineering Department in The University of Mosul.

\section{References}

Bau, H., Mcblane, G., \& Saferstein, I. (1983). Numerical Simulation Of Thermal Convection In An Eccentric Annulus Containing Porous Media. ASME 83 WA/HT 34.

Bejan, A. (1983). Natural Convection Heat Transfer In A Porous Layer With Internal Flow Obstructions. Int. J. Heat Mass Transfer, 26(6), 815-822. http://dx.doi.org/10.1016/S0017-9310(83)80105-7

Bejan, A. (1995). Convection Heat Transfer. New York, USA: John Wiley \& Sons, Inc.

Bishop, F. H., \& Charley, C.T. (1966). Photographic Of Natural Convection Between Concentric Cylinders (pp. 63-78). Proc., Heat Transfer Fluid Mechanics Institute, Stanford, CA: Stanford Univ. Press.

Braga, E. J., \& de Lemos, M. J. S. (2006). Simulation of Turbulent Natural Convection In A Porous Cylindrical Annulus Using A Macroscopic Two-Equation Model. Int. J. Heat Mass Transfer, 49, 4340-4351. http://dx.doi.org/10.1016/j.ijheatmasstransfer.2006.04.032

Caltagirone, J. P. (1976). Thermoconductive Instabilities In Porous Medium Bounded by Two Concentric Horizontal Cylinders. J. Fluid Mech., 76, 337- 362. http://dx.doi.org/10.1017/S0022112076000669

Charrier, M. C., Mojtabi, A., Azaiez, M., \& Labrosse, G. (1991). Numerical And Experimental Study Of Multicellular Free Convection Flows In An Annular Porous Layer. Int. J. Heat Mass Transfer, 34(12), 3061-3074. http://dx.doi.org/10.1016/0017-9310(91)90076-Q

Facas, G. N. (1994). Reducing the Heat Transfer From a Hot Pipe Buried in a Semi-infinite, Saturated, Porous Medium. J. Heat Transfer, 116, 473-476. http://dx.doi.org/10.1115/1.2911420

Hadim, H. \& North, M. (2005). Forced Convection in a Sintered Porous Channel with Inlet and Outlet Slots. Int. J. Thermal Sciences, 44, 33-42. http://dx.doi.org/10.1016/j.ijthermalsci.2004.04.016

Hoffmann, K. A., \& Chiang, S. T. (2000). Computational Fluid Dynamics I. Wichita, Kansas, USA: A Publication of Engineering Education System.

Kaviany, M. (1986). Non-Darcian Effects On Natural Convection In Porous Media Confined Between Horizontal Cylinders. Int. J. Heat Mass Transfer, 29(10), 1513-1519. http://dx.doi.org/10.1016/0017-9310(86)90066-9

Mota, J. P. B., \& Saatdjian, E. (1995). Natural Convection In Porous Cylindrical Annuli. Int. J. Num. Meth. Heat Fluid Flow, 5, 3-12. http://dx.doi.org/10.1108/EUM0000000004054

Mota, J. P. B., Esteves, I. A. A. C., Portugal, C. A. M., Esperança, J. M. S. S., \& Saatdjian, E. (2000). Natural 
Convection Heat Transfer In Horizontal Eccentric Elliptic Annuli Containing Saturated Porous Media. Int. J. Heat Mass Transfer, 43, 4367-4379. http://dx.doi.org/10.1016/S0017-9310(00)00068-5

Petrovic, Z., \& Stupar, S. (1996). Computational Fluid Dynamics I. Mech. Eng. Faculty, University Of Belgrade.

Saatdjian, E., Lam, R., \& Mota, J. P. B. (1999). Natural Convection Heat Transfer In The Annular Region Between Porous Confocal Ellipses. Int. J. Numer. Meth Fluids, 31, 513-522. http://dx.doi.org/10.1002/(SICI)1097-0363(19990930)31:2<513::AID-FLD889>3.0.CO;2-7

Sakr, R. Y., Berbish, N. S., Abd-Aziz, Ali A., \& Hanafi, A. S. (2008). Experimental and Numerical Investigation of Natural Convection Heat Transfer in Horizontal Elliptic Annuli. Journal of Applied Sciences Research, 4(2), 138-155.

Vafai, K. (2005). Handbook of Porous Media (2nd ed.). Taylor \& Francis Group, USA: CRC Press.

Zhu, Y. D., Shu, C., Qiu, J., \& Tani, J. (2003), Numerical Simulation of Natural Convection between Two Elliptical Cylinders Using DQ Method. Int. J. Heat Mass Transfer, 47(2004) 797-808. http://dx.doi.org/10.1016/j.ijheatmasstransfer.2003.06.005

\section{Copyrights}

Copyright for this article is retained by the author(s), with first publication rights granted to the journal.

This is an open-access article distributed under the terms and conditions of the Creative Commons Attribution license (http://creativecommons.org/licenses/by/3.0/). 